\title{
The Trust Degree Evolution of Public Opinion Propagation in a Complex Network
}

\author{
Xiangling Kuang ${ }^{1,2, *}$, Guangqiu Huang ${ }^{1}$, Lijun Yang ${ }^{2}$, Yuqing Yuan ${ }^{2}$, Xueqin $\mathrm{Li}^{1,3}$ and Lixia Cao ${ }^{1,4}$ \\ ${ }^{1}$ School of Management, Xi'an University of Architechture and Technology, Xi'an 710055, China \\ ${ }^{2}$ School of Economics and Management, Hubei University of Automotive Technology, Shiyan 442002, China \\ ${ }^{3}$ Data and Information Center, Xi'an Jiaotong University, Xi'an 710049, China \\ ${ }^{4}$ School of Science, Xi'an Technological University, Xi'an 710032, China
}

\begin{abstract}
A multi-community complex trust network evolution model is constructed and the fact that the minus value between the reward range and punishment range affects the trust degree of a complex trust network is made certain for the problem that the evolution of the trust weight value of the complex trust network being seldom considered. Firstly, a directed and weighted multi-community complex trust network model is built. Secondly, a new search algorithm of Poisson random walk visitor and a new model of propagation style with distinct attitude values of public opinion are established. Thirdly a method is designed for a node to select an opinion attitude value in accordance with the maximum trust value when two contradict attitude values are propagated to the node. Fourthly, the trust values of propagators are rewarded or punished after propagation. Finally, the model's simulation is carried on for several times and the trend in the evolution of trust values is analyzed for each time. The results show the trust values are mainly affected by the minus value between the reward range and punishment range. When the minus value is larger, the mean trust values of the communities and the whole net are bigger and vice versa. If the minus value is zero, the mean trust values trend to be ups and downs. The study considers the propagation not only in the opposite attitude values, but also based on the trust, beyond the propagation characteristics of public opinion considered by other researchers. The results are in good agreement with the actual situation.
\end{abstract}

Keywords: Complex network, trust network, opinion propagation, trusts degree.

\section{INTRODUCTION}

The trust degree between two people changes dynamically due to the opinion propagation in real life. If the public opinion is reliable, the trust degree between two communicators will continue to increase, otherwise it will continue to decrease. It is based on this fact that we need to study the evolution of the trust degree of public opinion propagation in a complex network.

Currently, many scholars have proposed trust models and studied values of the trust degree on trust models, influencing factors of the trust degree, evaluation of the trust degree in actual systems, transmission and propagation of the trust degree, etc.. Some scholars use trust models in service computing [1-3], e-commerce [4], virtual communities [5] and other fields [6,7], but they focus mainly on building methods of trust models and their effectiveness. In the field of public opinion propagation in complex network, the main concern is the evolution of public opinion propagation. The trust degree evolution of public opinion propagation in a complex network is not raised. There are also a number of scholars to study the trust degree evolution in supply chain management whose research methods are mainly social survey methods [8]. In this paper, we construct a directed and weighted complex network contained four communities, then the opposite opinion attitudes are spread on the network at the same time. When two opposite attitudes are spread to the same node, the opinion attitude value of the node will be updated according to the trust degrees between the two nodes. After each round of spread, the correct attitude of public opinion is announced. And then the five trust degree values of four communities and the whole network are checked according to the correct attitude, while rewards and punishments are implemented. Finally, the five trust degree evolution courses affected by public opinion propagating are examined.

\section{MODELING OF A PUBLIC OPINION PROPA- GATION METHOD IN A COMPLEX TRUST COMMUNITY NETWORK}

\subsection{Modeling of a Complex Trust Community Network}

In Reference [9], an unweighted and undirected complex community network is constructed. Based on it, we add trust 
values and directions to construct a directed weighted complex trust network A. In the trust network, the trust relationship between two users is usually described as: "user i trusts user $\mathrm{j}$ in some degree". Usually, $\mathrm{i}$ trusts $\mathrm{j}$ does not mean that $\mathrm{j}$ trusts $\mathrm{i}$, so the trust relationship is unequal. Trust relation in the corresponding network graph is indicated by a directed connecting edge from node $i$ to node $j$, as shown in Fig. (1). The weight value of the directed edge shows the trust degree between user $i$ and user $\mathrm{j}$ in time $t$. It is called trust value and denoted by $x_{i j}[10]$. In order to observe the continuous change of the trust value, we set the trust value to a nonnegative continuous change and no upper-limit section, that is $x_{i j}>0$. Trust value takes the random variable value $x_{i j}, x_{i j}>0, i>0, j>$ of function $\Gamma$. Its probability density function is

$$
p\left(x_{i j} ; \lambda, \mu\right)=\Gamma(\lambda, \mu)=\frac{\mu^{\lambda}}{\Gamma(\lambda)} x_{i j}^{\lambda-1} e^{-\mu x_{i j}} .
$$

When $\lambda$ and $\mu$ take different range values, the density curve of function $\Gamma$ has a different shape and it forms a special distribution, such as an exponential distribution, the chisquare distribution, normal distribution and Erlang distribution [11], so it has better adaptability. So the trust degree obeys $\Gamma$ distribution is better in actual running-in ability than to obey a simply distribution function.

\subsection{Modeling of a Public Opinion Attitude Propagation Process}

In reality, for one public opinion, different people have different views; attitudes of public opinion may be different. Usually people spread out their attitudes toward a pubic opinion when they disseminate the public opinion, so that you may be a person who receives different attitudes from different persons which are about the same public opinion. In response to this phenomenon, we design the following communication process of the public opinion attitude.

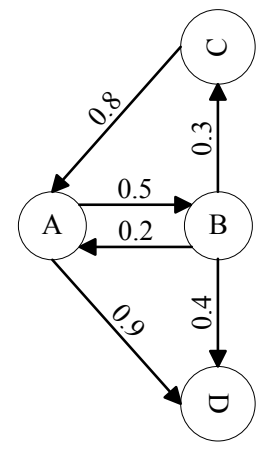

Fig. (1). A directed trust network.

The source nodes which spread public opinion attitudes are more than one node. Not each node likely to receive public opinion attitude is the neighbor of the source nodes. When a node receives several different attitudes to public opinion on the same event coming from different nodes, it needs to make clearly its attitude by determining how to choose the attitude of public opinion. In order to discuss the influence of public opinion attitudes towards people trust, so as to facilitate us to measure the communities and the overall trust level, we determine the attitude value of public opinion of the current node based on the trust value between nodes. Therefore, the propagation process model of public opinion is divided into three steps. The first step is to select several spread source nodes by random, and then randomly to assign to each source node different initial public opinion attitude value (shorter form is attitude value). The second step is to searches for its neighbor nodes to select the object node to be propagated by Poisson walker random walk search algorithm, which short form is PWRW and is designed by us. The third step is to propagate in accordance with model of susceptible infected removed attitude (the short form is SIRA), which is designed by us, and to assignment attitude values to the selected nodes. Then we loop the second step until all nodes have the attitude value.

In order to keep track of the search, each node is divided into three states, $\mathrm{S}$ state, I state and $\mathrm{R}$ state. Each state is stored in one array. $\mathrm{S}$ state represents a situation that a node dose not own attitude value yet. I state represents a situation that a node has hold an attitude value, but not all of its neighbors have hold attitude values. $\mathrm{R}$ state represents a situation that a node has hold an attitude value, and all of its neighbors have hold attitude values. In the directed weighted complex community trust network, after select multiple source nodes in accordance with the principle of random selection, the algorithm assign different initial attitude values $a$ and $b(a, b \in Z$ and $a \neq b)$ to the multiple source nodes, and put all source nodes into the array I to save, while put the other nodes into the array $\mathrm{S}$ to save. Then the algorithm searches the entire network following a method of Poisson walker random walk PWRW. If all the nodes are be visited, that is array S is empty, the algorithm terminates. When the source node $s_{i}$ applications PWRW strategy to spread attitude value $a$ in the network nodes, the node $s_{i}$ queries firstly the multiple neighbor nodes $t_{j}\left(j=1, \ldots m, 1 \leq m \leq L\left(t_{j}\right)\right.$. The value $L\left(t_{j}\right)$ is the sum number of neighbor nodes of the node $t_{j}$, which are found randomly following Poisson distribution. Then the node selects randomly the propagation order. Whether the node $t_{j}$ has its attitude value, it can spread the attitude value $a$ of the node $s_{i}$. If the node $t_{j}$ has no attitude value, then the attitude value $a$ of the node $s_{i}$ is spread to it, and its source node is recorded as the node $s_{i}$. If the node $t_{j}$ has the attitude value $a$ already, no spreading. If the node $t_{j}$ has the attitude value $b, b \neq a$ already, the node compares the trust degree between $d_{t s}\left(d_{t s}\right.$ is the trust degree of $t_{j}$ to $s_{i}$ ) and $d_{t r}$ ( $d_{t r}$ is the trust degree of $t_{j}$ to $r$, the previous node to spread attitude value $b$ to $t_{j}$ ) to determine to receive whose attitude value. The model of receive 
attitude values is called SIR-A. When $d_{t s}>d_{t r}, t_{j}$ receives the attitude value $a$ of the node $s_{i}$, and records $s_{i}$ as the source node who transmits the attitude value to it. When $d_{t s} \leq d_{t r}$, the node $t_{j}$ maintains the original attitude value $b$, not modifying the source node of it. After a round of propagation, all nodes in array $\mathrm{S}$ are checked. If a node already has the attitude value, it is moved to array I and is ready for the next round of the spread. All nodes in array I are checked. If all neighbors of a node have attitude value, then it is moved into array $\mathrm{R}$. Continue to spread in the next round until the array $\mathrm{S}$ are empty, then the algorithm ends.

The entire propagation process of SIR-A model can be simply represented by the following relationship formula (1):

$$
\left\{\begin{array}{l}
I_{a}(s)+S(t) \rightarrow I_{a}(s)+I_{a}(t) \\
I_{a}(s)+I_{a}(t) / R_{a}(t) \rightarrow I_{a}(s) / R_{a}(s)+I_{a}(t) / R_{a}(t) \\
I_{a}(s)+I_{b}(t) / R_{b}(t) \rightarrow I_{a}(s) / R_{a}(s)+I_{a}(t) / R_{a}(t) \\
\text { when } a \neq b \text { and } d_{t s}>d_{t r} \\
I_{a}(s)+I_{b}(t) / R_{b}(t) \rightarrow I_{a}(s) / R_{a}(s)+I_{b}(t) / R_{b}(t) \\
\text { when } a \neq b \text { and } d_{t s} \leq d_{t r}
\end{array}\right.
$$

\subsection{Modeling Trust Degree Calibration}

After a round of random propagation of public opinion in dissimilarity attitude values $a, b$, the nodes $t_{i}(i=1, \ldots N)$ that have passed public opinion attitude values need to be reward and punishment. Firstly, the algorithm generates randomly a final correct public opinion attitude value $T, T=a$ or $T=b$, and releases the final value $\mathrm{T}$ in the network. Secondly, the algorithm searches the attitude value $A, A=a$ or $A=b$ of each node $t_{i}(i=1, \ldots N)$ in order, and identifies their source nodes $s_{i}(i=1, \ldots N)$. If the attitude value of the current node and the right attitude values $T$ are the same, that is $A=T$, the trust value $d_{t s}$ of the current node's source nodes $s_{i}$ is rewarded. The reward rule is that the original trust value $d_{t s}$ is multiplied by a reward value $t P, 1 \leq t P \leq t P, 1 \leq t P \leq 2$, that is $t P, 1 \leq t P \leq 2 . d_{t s}=d_{t s} * t P$. If the attitude value $A$ of the current node and the right attitude values $T$ are different, that is $A=-T$, the trust value $d_{t s}$ of the current node's source nodes $s_{i}$ is punished. The punish rule is that the original trust value $d_{t s}$ is multiplied by a punish value $f P, 0<f P \leq 1 \quad t P, 1 \leq t P \leq 2 \quad$, that is $t P, 1 \leq t P \leq 2$ $d_{t s}=d_{t s} * f P$, as show in formula (2). Thirdly, the algorithm makes separate statistic of the mean trust values of each community and the entire network, in order to examine the impact to trust values of the spread of public opinion attitude value for communities and the whole network.

$d_{t s}=\left\{\begin{array}{l}d_{t s} * t P, 1 \leq t P \leq 2 \text { when } A=T \\ d_{t s} * f P, 0<f P \leq 1 \text { when } A=-T\end{array}\right.$
The whole process runs repeatedly several times, and finally takes out the mean trust values of the four communities and the whole network to make figures, showing the evolution of the process of trust values, and analysis of factors affected the trust values.

\section{EXPERIMENT AND SIMULATION}

In order to get how the public opinion propagation affects the trust values in the directed weighted complex communities trust network, we conducted simulation experiments based on the above model. We program the above model by using software MATLAB and make multi-step simulations, with Windows XP Professional 2002 as the platform, CPU is i3-2350 M, 2.3 GHZ, and memory is $3 \mathrm{~GB}$. The simulation process is: Firstly, it constructs a directed weighted complex community trust network. Secondly, it sets the simulation steps to 300 , and in each step carries out propagation of 40 original source nodes which have different public opinion attitude values in the whole network, in which the ratio of the positive public opinion attitude values and the total number of nodes is called positive ratio. If there are conflict attitude values in the propagationprocess, the algorithm takes one of the attitude values in accordance with the certain rules. Thirdly, the algorithm promulgates the right attitude value and carries on reward or punish to the trust value in the whole network. The reward parameters is $t P, 1 \leq t P \leq 2$. The $t P$ is taken 7 values include $1,1.2,1.3,1.5,1.8,1$ and 2 . The punish parameters is $f P, 0<f P \leq 1$. The $f P$ is taken 5 values include $0.2,0.5,0.6,0.8$ and 1 . After 300 steps, we observe changes of the average trust attitude values in each community and of the whole network. Repeating the test, each time we take different combination of reward parameter, punish parameter and positive ratio, so as to examine the effects of reward and punishment to the trust values.

\subsection{The Experimental Procedure}

Step 1: Firstly, the algorithm establishes a directed weighted complex community trust network. The network consists of 1024 nodes, 4 communities and in each community there are 256 nodes. The network map is stored with an adjacency matrix. The connection probability within the community is $p_{\text {in }}=0.995$, and that between the communities is $p_{\text {out }}=0.005, p_{\text {out }}<<p_{\text {in }}$. Secondly, it generates 4 intranet networks within each community respectively in accordance with their intranet network probabilities, and then links 4 communities in accordance with their interconnection network probability. Thirdly, it assigns values to the trust degrees in whole network. In the trust function $\Gamma$, let $\lambda=\mu=1$ , that is, the initial trust values are taken in range $(0,+\infty)$, but mostly lie nearby 1 . Because we inspect mainly the changes of trust values, and they are the minus values of subsequent values relative to the initial values, the initial values are only reference values for the change, and they themselves do not affect the results. Every node's number 
Table 1. Simulation parameter settings and the results table of $\mathbf{3}$ typical simulation experiments.

\begin{tabular}{|c|c|c|c|c|c|c|c|}
\hline Group & tp & fp & tp-1-(1-fp) & Opratio & Trend of the Lines & $\begin{array}{c}\text { Trust Average Value of the } \\
\text { Whole Network }\end{array}$ & Figure No. \\
\hline \hline 1 & 1.3 & 0.5 & -0.2 & 0.8 & 4lines are all down & 0.6945 & Fig. (2) \\
\hline 2 & 1.2 & 0.8 & 0 & 0.3 & a little up or flat & Fig. (3) \\
\hline 3 & 1.5 & 1 & 0.5 & 0.5 & 4lines are all up & 12385.76 & Fig. (4) \\
\hline
\end{tabular}

ID, trust degree, attitude value, and all the neighbor nodes are stored in a cell data structure.

Step 2: The algorithm initializes network public opinion attitude values. Firstly, the algorithm initializes the data structure, which is node cell, used to store each node's attitude value and neighbor nodes. Secondly, it selects randomly 40 nodes in 1024 nodes as the first to know public opinion throughout the network nodes. Their attitude values of public opinion are discrete to +1 or -1 , according to total three kinds of probability values $0.3,0.5$ and 0.8 , as the initial diversity attitude values of the source node. Three kinds of probabilities are called the initial positive ratio opratio, which means the proportion that the number of nodes whose public opinion attitude values are +1 to the number of the initial 40 nodes. Thirdly, it initializes three arrays: unKnown, liveKnown and deadKnow arrays, which are S state, I state, and $\mathrm{R}$ state node array. They are used to store the nodes which have not received public opinion in current, have public opinion attitude values and owned the spreading capability, and have public opinion attitude values but not have the spreading capability.

Step 3: The algorithm conducts a round of public opinion attitude value propagation. Firstly, it traverses all nodes in the liveKnown set, identifies all subsequent nodes of current nodes, determines several object nodes to be propagated by Poisson distribution, and generates candidate node sequences by randomly selection. Secondly, the algorithm sequentially spreads attitude values to candidate nodes. It checks whether the candidate node has an attitude value or not. If the candidate node has no attitude value, the spreading node ID is written as the transmission source node to the data structure of public opinion attitude value receiver, and the attitude value of the receiver is set to the value of the source node. If the candidate node has an attitude value, the algorithm compares the trust value of the candidate node to the preview source node and the trust value to the current node. If the trust value to the current node is larger, the attitude value of the candidate node is modified to the attitude value of the current node, the subsequent node of the source node is updated, whereas no changes. Continue to traverse the candidate node set until all candidate nodes are checked. Thirdly, the algorithm checks the unKnown set. If the node has already held views, it is moved to the liveKnown set. It checks the liveKnown set. If all the neighbors of a node are already held views, the node is moved to the deadKnown set. Continue to traverse the nodes in the liveKnown set until it is empty, that is, all nodes currently can be spread outwardly is checked. Continue to traverse the nodes in the unKnown set until it is empty, that is, there is no node which has no attitude value currently, and this round propagation is end. Otherwise, repeat step 3 .

Step 4: The algorithm implements a round of reward and punish to trust degree. After the end of public opinion spreading, it randomly generates finally correct public opinion attitude value +1 or -1 . After we input a reward value to parameter $t P$ and a punishment value to parameter $f P$, it traverses all the nodes in the network to compare whether their attitude values and the correct attitude value are the same. If the same, the trust value of current node to the source node is multiplied by the reward parameter to adjust. If inconsistent, the trust value of current node to the source node is multiplied by the punish parameter to adjust.

Step 5: The algorithm computes 4 communities and overall trust indicators. It sets 5 set of indicators to quantify the average trust in the network. Four groups are stored the average trust values of all sides in four communities, while one group is stored the average trust value of all sides in the entire network. After completing reward and punish trust values, it makes a statistics of the trust degree index with the adjacency matrix. It judges whether public opinion is spread to 300 times. If it is, the program ends. It imports the 300 time trust data of 4 communities and the overall network to EXCEL file to make statistics. Otherwise, return to step 2.

\subsection{Analysis of experimental results}

In this study, we conduct totally 36 times simulation experiments. But consider of the given space, we just list three typical examples in them. In each experiment with different reward parameter $t P$, punish parameter $f P$, positive ratio opratio, and all the propagation times are set to 300 steps, the simulation detects how public opinion propagation influences the trust values. The corresponding values of the selected parameters and their results are shown in Table $\mathbf{1}$. Finally, all the calculated trust degree indicators are stored in EXCEL files, and each corresponding graph is drawn with software origin, specifically as follows.

(1) When the minus of two amplitude values of reward and punish parameter is less than zero, i.e., tp-1- (1-fp) $<0$, the overall trust trend lines are down totally, that is, whether it is the average trust value of the community or of the whole network, it is declining. In Table $\mathbf{1}$ it is represented by group 1. Specific trend is shown in Fig. (2). 


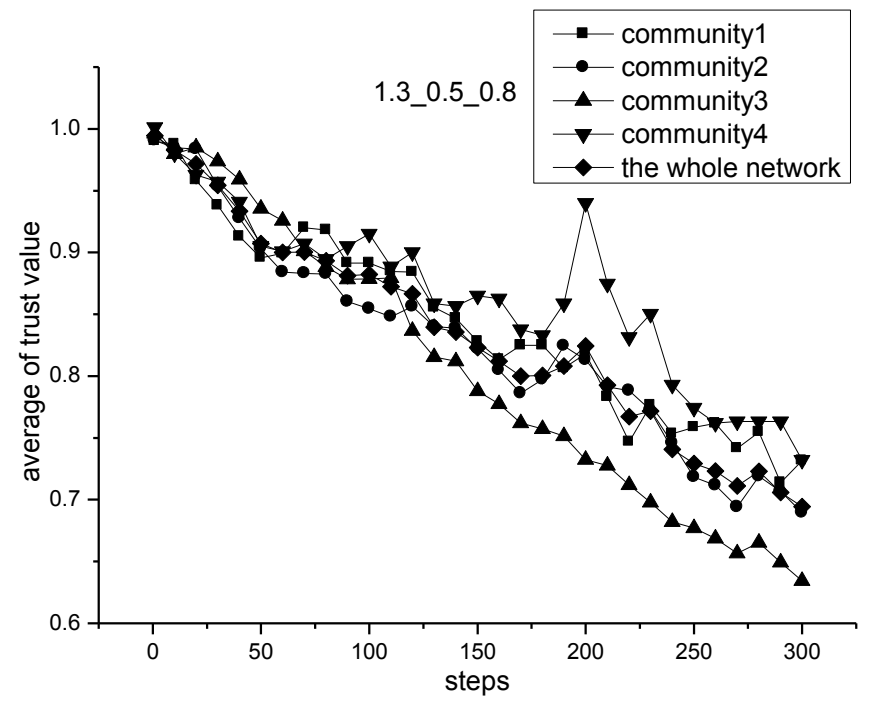

Fig. (2). Simulation results (tp: 1.3, fp: 0.5, opratio: 0.8).

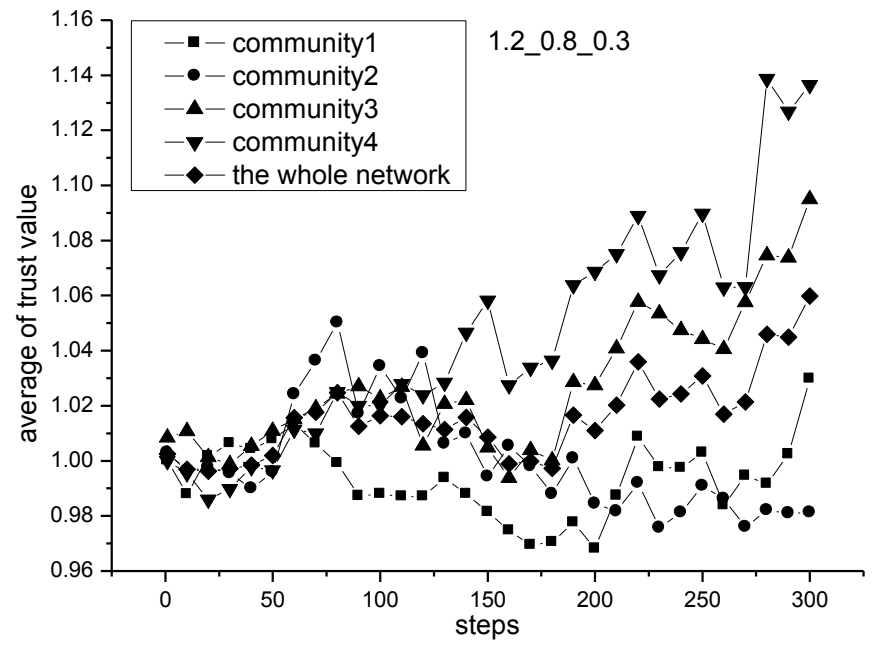

Fig. (3). Simulation results (tp: 1.2, fp: 0.8, opratio: 1).

(2) When the minus of two amplitude values of reward and punish parameter is equal to zero, i.e., tp-1- $(1-\mathrm{fp})=0$, the overall trust trend lines are rise or fall. In Table $\mathbf{1}$ it is represented by group 2. Specific trend is shown in Fig. (3). When a parameter has a little increase or decrease the ratio, the impact on the trust value is not obvious. But when the increase or decrease part is large, trust values start to vibrate turbulently in some communities. We analyze this vibration is generated based on the randomness of the experiment. Because when the public opinion attitude values are initialized, it is based on a certain probability to randomly assign to 40 nodes attitude values. This may probably cause that the 40 node points are mostly located in one community, or their initial values are taken too much only one value. Although the probability is small, if in this situation the final public opinion attitude value given randomly by the algorithm is equal to the initial value, the trust degree of the entire network will be a sudden increase, whereas the mass decrease.

(3) When the minus of two amplitude values of reward and punish parameter is greater than zero, i.e., tp-1- (1-fp) $>0$, the overall trust trend lines are up totally, that is, whether it is the average trust value of the community or of the whole network, it is on the rise. In Table $\mathbf{1}$ it is represented by group 3. Specific trend is shown in Fig. (4).

When the minus value is getting bigger, the upward trend of trust value is accelerated and ultimately mean values are very large. Figs. (2) and (4) are in good agreement with the reality.

(4) The initial positive ratio does not affect the trust value trend. We set 3 positive ratio parameters $(0.3,0.5,0.8)$ to six different groups parameter, find rising or falling trends of 


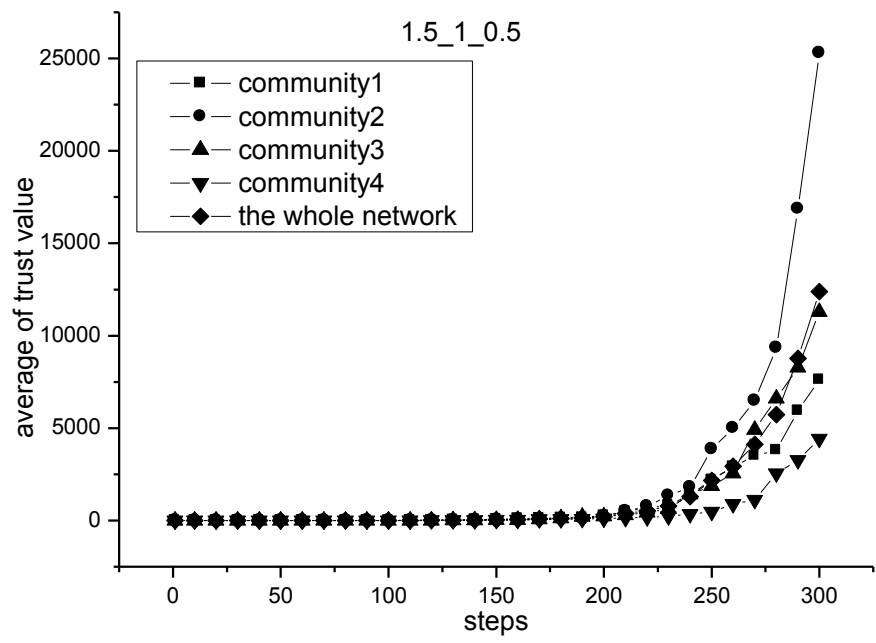

Fig. (4). Simulation results (tp: 1.5 , fp: 1 , opratio: 0.5 ).

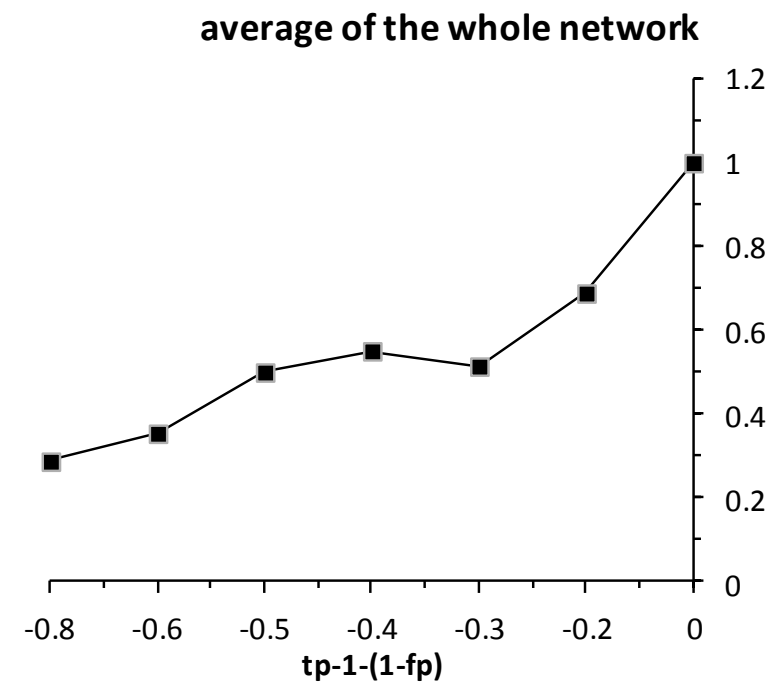

Fig. (5). The relationship figure of the minus value of two amplitude values of reward and punish parameter to the trust value of the whole network when the minus value is negative.

trust values are not affected, and find the average trust value of the whole network has no correlativity with the positive ratio. But when the minus of two amplitude values of reward and punish parameter is zero, due to the overall trend line ups and downs and the trust trend is random, the initial positive ratio affects the shape of the trust value lines.

(5) The structure of communities does not affect the movements of final trust lines in the propagation process of public opinion attitude value, and it dose only affect the speed of operation. When the connecting probability in a community is large, and community connects tightly, the propagating velocity of public opinion attitude values in community is relatively quick. When the connecting probability in a community is small, and community connects not such tightly, the propagating velocity of public opinion attitude values in community is relatively slow. In the situation of the same reward and punish parameters, the greater the probability that the community connection, the higher trust value of the final value in the whole network is.
(6) Comparing the minus value of two amplitude values of reward and punish parameter with the average trust value of the whole network, we further analyze the relationship between them, as shown in Figs. (5) and (6). When the minus value is negative, the smaller the value is, the smaller the average trust value of whole network is. When the minus value is 0 , the average trust value of the whole network reaches 1 . When the minus value is positive, the larger the value is, the larger the average trust value of whole network is.

\section{CONCLUSION}

In this paper, we construct a directed weighted complex trust network, on which we conduct a group of simulation experiments of propagation in diversity public opinions nodes, so as to examine how public opinion's propagation affects the trust degree on the network. When a node receives two different public opinion attitude values, we 


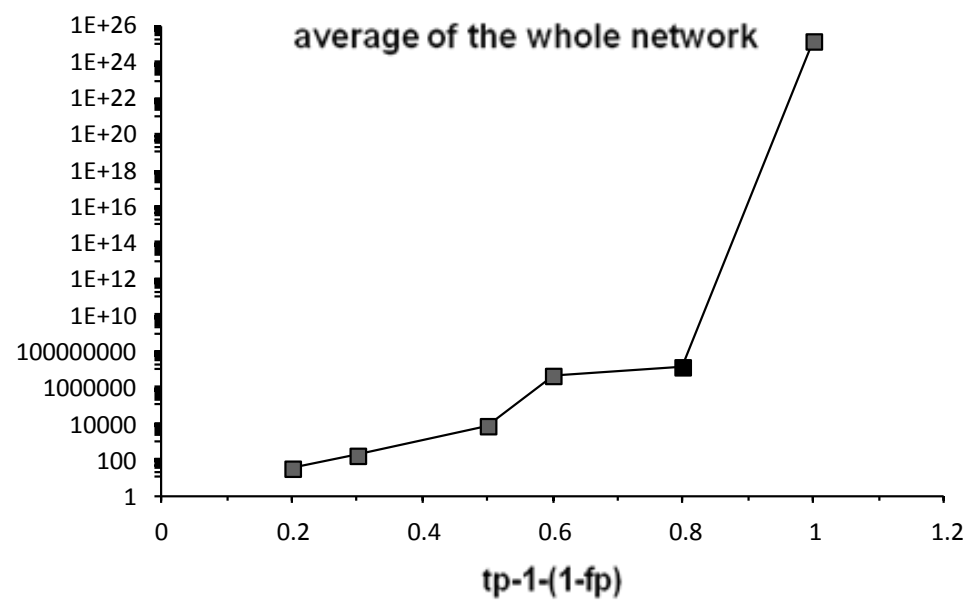

Fig. (6). The relationship figure of the minus value of two amplitude values of reward and punish parameter to the trust value of the whole network when the minus value is positive. (The vertical axis is logarithmic scale).

process it with maximum trust algorithm. Then we conduct a number of simulations for the algorithm to achieve the evolution analysis of the trust value in the process of the public opinion propagation in complex trust network, and obtain a few main points about how the minus value of two amplitude values of reward and punish parameter affects the trust value. They confirm the effects of public opinion propagation to complex trust network, and agree with the effect rules of propagation. It shows that the main reason of a raise trend of the trust degree in real social network is probably that the reward to right message communicators is greater than punishment to error message communicators. The main reason of a decline trend of the trust degree is probably that the reward to right message communicators is less than the punishment to error message communicators.

\section{CONFLICT OF INTEREST}

The author confirms that this article content has no conflict of interest.

\section{ACKNOWLEDGEMENTS}

This work is supported partly by the Humanity and Social Science Foundation of Education Department of Hubei Province (No. 13q088), Humanity and Social Science Foundation of Ministry of Education of China (No: 14YJA630079) and Doctoral Foundation of Hubei University of Automotive Technology (No. BK201203).

\section{REFERENCES}

[1] J. Long, X. M. Liu, X. P. Yuan, Z. P. Zhang, and W. H. Gui, "A Web Services Composition Strategy Based on Trust Reasoning and Evolution", Chinese Journal of Computers, vol. 35, pp. 298314, Feb. 2012.

[2] G. J. Oyewole and A. E. Oluleye, and E.O. Oyetunji, "Minimizing Maximum Stretch on a Single Machine with Release Dates", $A d-$ vances in Industrial Engineering and Management, vol. 3, no. 1, pp. 1-12, 2014. doi:10.7508/AIEM-V3-N1-1-12

[3] L. Xin, Y. S. Fan, X. Li, and N. Y. Yu, "QPrefR: A Preferenceaware Recommendation Scheme Based on Service Reputation", Computer Integrated Manufacturing System, vol. 6, pp. 210-230, June 2013.

[4] X. F. Xia, Y. Li, and L. H. Cong, "Construction of Customer Trust Network for Web Viral Marketing", Journal of Chinese Computer Systems, vol. 34, pp. 2075-2080, Sep. 2013.

[5] M. H. Qian and C. Wang, "Research Progress and Enlightenment of Online Trust in Virtual Community", Inquiry into Economic Issues, pp. 156-161, May 2013.

[6] W. J. Wang and D. W. Wang, "Survey on Online Auction Trust Models", Computer Engineering and Applications, vol. 46, pp. 15, Oct. 2010

[7] X. P. Hu and J. Yin, "A Review of Trust Transitivity Models", Journal of Southeast University (Philosophy and Social Science), vol. 15, pp. 46-51, Jul. 2013.

[8] Z. H. Xie, "Research on Operation Mechanism of Collaboration Trust in Automobile Manufacturing Supply Chain", Tianjin: University of Tanjin, 2010.

[9] Grabowski A and Kosin ski R A, "Epidemic Spreading in A Hierarchical Social Network", Physical Review E, vol. 73, no. 3, 032908, 2004.

[10] J. Wang, Y. H. Liu, and Y. Jiao, "Model for Trust Propagation in Web", Computer Engineering and Applications, vol. 45, pp. 136138, July 2009.

S. S. Mao and J. X. Zhou, "Probability Theory and Mathematical Statistics", Beijing: China Statistics Press, 2000, pp. 90-93.

Received: September 16, 2014

(C) Kuang et al.; Licensee Bentham Open.

This is an open access article licensed under the terms of the Creative Commons Attribution Non-Commercial License (http://creativecommons.org/licenses/by-nc/3.0/) which permits unrestricted, non-commercial use, distribution and reproduction in any medium, provided the work is properly cited. 